Fabio Franzese \& Ingmar Rapp

\title{
Der Einfluss von Arbeitslosigkeit auf das Trennungsrisiko von Ehen
}

\author{
The impact of unemployment on the risk of marital separation
}

\begin{abstract}
Zusammenfassung:
Der Beitrag untersucht den Zusammenhang zwischen Arbeitslosigkeit und der Stabilität von Ehen. Dabei werden auch Veränderungen dieses Zusammenhangs seit Mitte der 1980er Jahre sowie die Bedeutung der Dauer der Arbeitslosigkeit untersucht. Datengrundlage ist das Soziooekonomische Panel (SOEP). Die Ergebnisse zeigen, dass Arbeitslosigkeit eines Partners, v. a. des Mannes, auch unter Kontrolle der beruflichen Ausbildung sowie des Einkommens, das Trennungsrisiko von Ehen erhöht. Bei Männern ist sowohl bei kurzer als auch bei längerer Dauer der Arbeitslosigkeit ein erhöhtes Trennungsrisiko zu beobachten, wobei das Trennungsrisiko bei einer Dauer von einem bis zwei Jahren am höchsten ist. Außerdem zeigt sich, dass der destabilisierende Effekt der Arbeitslosigkeit des Mannes im Zeitverlauf schwächer wird.
\end{abstract}

Schlagwörter: Trennung, Scheidung, Arbeitslosigkeit, Sozio-oekonomisches Panel

\begin{abstract}
:
This study analyzes the relationship between unemployment and the risk of marital separation. In addition, possible changes in the effect of unemployment since the mid-eighties as well as the duration of unemployment are examined. The data base is the German Socio-Economic Panel, which provides data from 1984 to 2010 . The results indicate a higher risk of separation if a spouse, especially the husband, is unemployed. In respect of the length of unemployment, it is shown that both short and longer periods of men's unemployment destabilize marriage. Highest risk of separation appears for men who are without a job for between one and two years. Furthermore, this study suggests that the influence of men's unemployment on the risk of separation is significantly lower in the period from 2000 to 2010 than in the late 1980 s.
\end{abstract}

Key words: separation, divorce, unemployment, German Socio-Economic Panel 


\section{Fragestellung}

Arbeitslosigkeit ist schon seit längerer Zeit eines der drängendsten gesellschaftlichen Probleme in den Industrieländern. Während die Arbeitslosigkeit in Deutschland in den letzten Jahren rückläufig war, erreicht sie momentan in einigen europäischen Ländern Höchststände (OECD 2012). Eine hohe Arbeitslosenquote ist auf gesamtgesellschaftlicher Ebene ein Problem, da sie Zeichen für eine schlechte Wirtschaftslage ist und für den Staat hohe Ausgaben zur Folge hat. Auch auf individueller Ebene geht Arbeitslosigkeit mit vielfältigen, zumeist unerwünschten Konsequenzen einher. Neben offensichtlichen Folgen, wie einer mehr oder weniger großen Reduktion des Einkommens (BMAS 2013), zählen hierzu zum Beispiel eine Verschlechterung der Gesundheit (Kessler et al. 1987; Weber et al. 2007) und der psychischen Verfassung (Aubry et al. 1990; Kessler et al. 1987). Auch geht Arbeitslosigkeit oft mit einer geringeren Zufriedenheit mit der Ehe (Atkinson et al. 1986; Aubry et al. 1990; Bleich/Witte 1992; vgl. aber auch Brinkerhoff/White 1978) sowie mit einer geringeren allgemeinen Lebenszufriedenheit (u. a. Kassenboehmer/Haisken-DeNew 2009; Gerlach/Stephan 1996, 2001; Winkelmann/Winkelmann 1998) einher.

An dieser Stelle wird untersucht, ob Arbeitslosigkeit das Trennungsrisiko von Ehen beeinflusst. Vorliegende Untersuchungen weisen darauf hin, dass Arbeitslosigkeit mit einem erhöhten Trennungs- und Scheidungsrisiko einhergeht (z.B. für Deutschland: Kraft 2001), wobei jedoch einige Fragen offen geblieben sind. Erstens stellt sich die Frage, ob Arbeitslosigkeit das Trennungsrisiko ursächlich erhöht, oder ob dritte Faktoren gleichzeitig sowohl Trennungen als auch Arbeitslosigkeit wahrscheinlicher machen. Zweitens ist bislang unklar, welche Faktoren den Einfluss von Arbeitslosigkeit auf das Trennungsrisiko möglicherweise vermitteln. Unklar ist auch drittens, wie sich das Trennungsrisiko mit zunehmender Dauer der Arbeitslosigkeit verändert. Viertens stellt sich die Frage nach Veränderungen des Einflusses von Arbeitslosigkeit auf das Trennungsrisiko im Zeitverlauf. Die vorliegende Untersuchung geht diesen Fragen nach und berücksichtigt hierbei auch mögliche Geschlechterunterschiede.

\section{Bisherige empirische Befunde zum Einfluss von Arbeitslosigkeit auf die Ehestabilität}

Bereits kurz nach der schweren Wirtschaftskrise in den USA in den 1930er Jahren, der sog. Great Depression, wurde der Einfluss von Arbeitslosigkeit der Ehemänner auf die familiären Beziehungen untersucht (Angell 1936; Bakke 1940; Cavan/Ranck 1939; Komarovsky 1940). Trotz methodischer Unzulänglichkeiten kamen alle diese Studien zu ähnlichen Ergebnissen. Arbeitslosigkeit des Mannes hat demnach negative Effekte auf das Selbstbewusstsein des Mannes sowie auf die Partnerschaft und auf die familiären Beziehungen (vgl. Larson 1984: 503). Die Studien aus den 1930er und 1940er Jahren weisen darauf hin, dass Arbeitslosigkeit ein Auslöser für familiäre Krisen ist (für eine Übersicht siehe Eisenberg/Lazarsfeld 1938).

$\mathrm{Zu}$ den ungünstigen Effekten von Arbeitslosigkeit, die in diesen und in späteren Studien nachgewiesen wurden, gehören Rollenkonflikte, eine schlechtere Kommunikation 
zwischen den Ehepartnern, eine geringere Qualität der Ehe und auch ein höheres Trennungs- und Scheidungsrisiko (Atkinson et al. 1986; Jensen/Smith 1990; Lampard 1994; Larson 1984; Starkey 1996; Powell/Driscoll 1973). Auch aktuellere Studien aus den USA (Charles/Stephens 2004, Lewin 2005), Großbritannien (Böheim/Ermisch 2001, Doiron/ Mendolia 2011), Finnland (Jalovaara 2001, 2003), Norwegen (Hansen 2005; Rege et al. 2007) und Schweden (Eliason 2012) kommen einheitlich zu dem Ergebnis, dass Arbeitslosigkeit einen destabilisierenden Effekt auf Ehen hat. ${ }^{1}$

Dies gilt auch für die beiden einzigen für Deutschland vorliegenden Studien. Cooke und Gash (2010) untersuchen für Westdeutschland die Auswirkungen von Teilzeitarbeit der Ehefrau auf das Trennungsrisiko. Dabei kontrollieren sie auch den Erwerbsstatus des Mannes. Arbeitslosigkeit des Mannes erhöht demnach das Scheidungsrisiko tendenziell, jedoch nicht signifikant (Cooke/Gash 2010). Als bislang einzige Studie befasst sich die Untersuchung von Kraft (2001) zentral mit der Auswirkung von Arbeitslosigkeit auf das Trennungsrisiko in Deutschland. Mit Daten des Sozio-oekonomischen Panels aus den Jahren 1987 bis 1996 kommt diese Studie zu dem Ergebnis, dass Arbeitslosigkeit das Trennungsrisiko signifikant erhöht. Dieser Effekt ist für Männer stärker als für Frauen und er ist umso größer, je mehr Monate eine Person im Vorjahr arbeitslos war.

\section{Erklärungsansätze und Hypothesen zum Einfluss von Arbeitslosigkeit auf die Ehestabilität}

Zur Erklärung der Ehestabilität wird häufig auf austauschtheoretische und familienökonomische Erklärungsmodelle zurückgegriffen. Austauschtheoretischen Überlegungen zufolge beruht die Ehestabilität in erster Linie auf der Ehequalität, d.h. auf der subjektiven Bewertung der ehelichen Beziehung (vgl. Levinger 1976, 1982; Lewis/Spanier 1979, 1982; Nye 1982). Darauf, dass Arbeitslosigkeit die Ehequalität reduzieren könnte, weisen Studien hin, die zeigen, dass Arbeitslosigkeit zu häufigerem Streit (Hess et al. 1991: 190; Larson 1984; Wilhelm/Ridley 1988; Zvonkovic et al. 1988) und zu Rollenkonflikten (Hess et al. 1991: 186; Cavan 1959; Schindler/Wetzels 1990; Hartley 1987) führen kann. Arbeitslosigkeit könnte demnach, vermittelt über häufigeren Streit und Rollenkonflikte, die Ehequalität negativ beeinflussen und somit das Trennungsrisiko erhöhen. Auf Grundlage von familienökonomischen Überlegungen muss man außerdem davon ausgehen, dass unerwartete Ereignisse, zu denen der Eintritt von Arbeitslosigkeit zählt, den „match“, d.h. wie gut die Partner zusammenpassen, verändern (Becker et a. 1977). Sofern Arbeitslosig-

1 Die Frage nach einem Zusammenhang von Arbeitslosigkeit und Ehescheidung wurde zum Teil auch in Makro-Analysen untersucht. In einer Studie über den Zusammenhang von Arbeitslosen- und Scheidungsrate in Japan im Zeitraum von 1958 bis 2006 konnte eine positive Korrelation festgestellt werden (Kawata 2008). In einer weiteren Makro-Analyse, in der die Bundesstaaten der USA untersucht wurden, fanden Amato und Beattie (2011) von 1960 bis 1980 einen positiven und nach 1980 einen negativen Zusammenhang. Kalmijn (2007) fand in einer Studie über insgesamt 37 europäische Länder in den 1990er Jahren Hinweise für einen negativen Zusammenhang von Arbeitslosen- und Scheidungsrate. Aus solchen Korrelationen auf Länderebene lässt sich aber nicht auf einen Effekt von Arbeitslosigkeit auf das Trennungsrisiko beim Individuum schließen. 
keit und der damit einhergehende Einkommens- und Statusverlust die Attraktivität des arbeitslosen Partners für seinen Partner reduziert, werden für diesen alternative Partner vergleichsweise attraktiver und Trennungen somit wahrscheinlicher. Hinzu kommt, dass durch den Eintritt in die Arbeitslosigkeit beim arbeitslosen Partner Eigenschaften sichtbar werden können, die dem anderen Partner vorher unbekannt waren, was ebenfalls zu einem Anstieg des Trennungsrisikos beitragen kann. Das in jüngerer Zeit vorgeschlagene Modell der Frame-Selektion (Esser 2002) vereint die rationale mit einer normativen Sichtweise auf Ehen. Es geht davon aus, dass Kosten-Nutzen-Kalküle für die Entscheidung über die Aufrechterhaltung der Ehe keine Rolle spielen, wenn diese durch einen „Frame“ als unzerbrechliche Beziehung gerahmt ist. Die genannten austauschtheoretischen und familienökonomischen Mechanismen wirken demnach nur bei Ehen, in denen die Unzerbrechlichkeit der Ehe kein Teil des Frames ist. Somit ist auch nach dem Modell der Frame-Selektion zu erwarten, dass Arbeitslosigkeit das Trennungsrisiko im Durchschnitt aller Ehen erhöht. Die genannten Argumente lassen somit einhellig erwarten, dass Arbeitslosigkeit das Trennungsrisiko erhöht (Hypothese 1a).

Diese Hypothese wird auch durch die in Kapitel 2 zusammengefassten Befunde aus früheren Studien gestützt. Nichtsdestotrotz sind weitere Überlegungen in Betracht zu ziehen. Erstens verweist die individual liabilities perspective (Amato/Beattie 2011: 707f.) auf eine alternative Erklärung für den vielfach beobachteten positiven Zusammenhang zwischen Arbeitslosigkeit und Trennungswahrscheinlichkeit. Demnach beruht der Zusammenhang zwischen Arbeitslosigkeit und Trennungsrisiko auf einer Scheinkorrelation (vgl. auch Kraft 2001: 74). ${ }^{2}$ Auslöser für beides, Arbeitslosigkeit und Trennung, sind in dieser Perspektive individuelle Eigenschaften einer Person, die sowohl vom Arbeitgeber als auch vom Ehepartner negativ bewertet werden. Als solche Eigenschaften kommen z.B. unsoziales Verhalten sowie Alkohol- und Drogenmissbrauch in Betracht. Zweitens gibt es Argumente dafür, dass Arbeitslosigkeit auch mit stabilisierenden Faktoren einhergehen kann. Ausgehend von austauschtheoretischen Überlegungen ist denkbar, dass es durch den Eintritt in die Arbeitslosigkeit eines Partners zur Erhöhung der Trennungsbarrieren kommt. Während der Verlust des eigenen Erwerbseinkommens die ökonomische Abhängigkeit des arbeitslosen Partners erhöht, fühlt sich dessen Partner möglicherweise verpflichtet, den Ehepartner gerade in schlechten Zeiten zu unterstützen. Zudem verfügen Arbeitslose über mehr Freizeit, was von einigen Arbeitslosen und ihren Familien positiv empfunden wird, da sie mehr Zeit mit dem Ehepartner und der Familie sowie mit Freizeitbeschäftigungen verbringen können (Brinkmann 1984; Zvonkovic et al. 1988). Einige Studien berichten, dass diese gute Seite der Arbeitslosigkeit eher von Frauen gesehen wird (Brinkmann 1976: 407 ff.; Hartley 1987; Root 1984). Schließlich könnte Arbeitslosigkeit eines Partners eine spezialisierte Arbeitsteilung von Erwerbs- und Hausarbeit begünstigen, was familienökonomischen Überlegungen zufolge die Ehe stabilisieren sollte (Becker 1993). Auf dieser Grundlage lässt sich die Alternativhypothese formulieren, dass Arbeitslosigkeit das Trennungsrisiko nicht erhöht (Hypothese 1b).

Die bis hierhin genannten Mechanismen geben Grund zu der Annahme, dass Arbeitslosigkeit von Männern und Frauen unterschiedliche Effekte auf die Ehestabilität haben könnte. Mögliche Geschlechterunterschiede im Einfluss des Erwerbsstatus auf das Trennungsri-

2 Empirische Hinweise hierfür liefert Lampard (1994: 296). 
siko könnten daraus folgen, dass - v.a. in älteren Kohorten - der Mann häufiger der Hauptverdiener ist und in diesem Fall der finanzielle Ausfall bei Arbeitslosigkeit der Frau weniger gravierend ist. Bei einer Orientierung am traditionellen Ein-Ernährer-Modell ist zudem zu vermuten, dass bei arbeitslosen Männern die Unzufriedenheit höher ist und Rollenkonflikte wahrscheinlicher sind als bei arbeitslosen Frauen. Gestützt wird diese Vermutung durch Ergebnisse von Brinkmann aus dem Jahr 1976, wonach arbeitslose Männer stärker unter psychosozialen Belastungen leiden als arbeitslose Frauen. In Bezug auf mögliche Geschlechterunterschiede ist daher zu erwarten, dass Arbeitslosigkeit des Ehemannes das Trennungsrisiko in stärkerem Maße erhöht als Arbeitslosigkeit der Ehefrau (Hypothese 2).

Als möglicher Vermittlungsfaktor für einen ungünstigen Einfluss von Arbeitslosigkeit auf das Trennungsrisiko kommt das infolge von Arbeitslosigkeit sinkende Einkommen in Betracht. Wie aus einer Studie von Dew (2011) hervorgeht, tragen vor allem Streitigkeiten über finanzielle Themen zu einem erhöhten Trennungsrisiko bei. Dieses Ergebnis steht im Einklang mit familienökonomischen und austauschtheoretischen Überlegungen, wonach ein durch Arbeitslosigkeit bedingter Einkommensverlust mit einer abnehmenden Ehequalität bzw. mit einem sinkenden Ehegewinn und folglich mit einem steigenden Trennungsrisiko einhergeht. Somit ist zu erwarten, dass der destabilisierende Effekt von Arbeitslosigkeit auf die Ehebeziehung zu einem Teil auf den mit Arbeitslosigkeit einhergehenden Einkommensverlust zurückzuführen ist (Hypothese 3).

Des Weiteren ist in Betracht zu ziehen, dass sich der Einfluss der Arbeitslosigkeit auf das Trennungsrisiko mit der Dauer der Arbeitslosigkeit verändert. Bereits unmittelbar nach Eintritt von Arbeitslosigkeit finden ein Wandel des täglichen Lebens sowie ein Einkommensverlust statt, was vor allem unmittelbar nach Eintritt der Arbeitslosigkeit zu Trennungen führen könnte. Demgegenüber sollten nach langer Arbeitslosigkeitsdauer nur noch wenige Trennungen stattfinden, weil die noch verbleibenden Ehen eine selektive Gruppe von Paaren darstellen, bei denen Arbeitslosigkeit offensichtlich nicht zur Trennung führt. Vor diesem Hintergrund ist zu erwarten, dass der Effekt von Arbeitslosigkeit auf das Trennungsrisiko mit zunehmender Dauer der Arbeitslosigkeit abnimmt (Hypothese 4a).

Andererseits ist auch in Betracht zu ziehen, dass Arbeitslosigkeit das Trennungsrisiko erst dann erhöht, wenn sie eine gewisse Zeit andauert. So kann eine kurze Arbeitslosigkeit auch freiwillig sein (Kraft 2001: 76). Was die mit Arbeitslosigkeit einhergehenden (z.B. finanziellen) Belastungen anbelangt, nehmen diese außerdem zu, wenn Arbeitslosigkeit länger andauert. Dasselbe gilt für den mit Arbeitslosigkeit einhergehenden Statusverlust. Auf dieser Grundlage ist denkbar, dass der ungünstige Effekt von Arbeitslosigkeit mit zunehmender Arbeitslosigkeitsdauer nicht abnimmt, sondern ansteigt (Hypothese 4b).

Mit Blick auf den Wandel der Geschlechterrollen ist schließlich in Betracht zu ziehen, dass sich die Auswirkungen von Arbeitslosigkeit auf das Trennungsrisiko im Zeitverlauf verändert haben. Während für Männer Erwerbsarbeit heute ebenso wie früher die Norm darstellt, wurde Erwerbstätigkeit für (Ehe-)Frauen erst in jüngerer Zeit zunehmend zur Regel, wodurch vermutlich auch die finanziellen und psychosozialen Belastungen für die Ehepartner im Falle einer Arbeitslosigkeit der Frau zu- und im Falle der Arbeitslosigkeit des Mannes abgenommen haben. Vor diesem Hintergrund ist zu erwarten, dass der destabilisierende Effekt von Arbeitslosigkeit in jüngerer Zeit für Männer abgenommen und für Frauen zugenommen hat (Hypothese 5). 


\section{Daten und Methode}

Um die Hypothesen über den Einfluss der Arbeitslosigkeit auf das Trennungsrisiko von Ehepaaren zu überprüfen, werden Daten des Sozio-oekonomischen Panels (SOEP) verwendet (vgl. Wagner et al. 2008). Es handelt sich dabei um eine für Deutschland repräsentative Wiederholungsbefragung, die seit 1984 in einjährigem Rhythmus durchgeführt wird. Aufgrund der langen Zeitspanne des Panels eignen sich die Daten gut für Analysen der Ehestabilität. Ein weiterer Vorteil der Daten ist, dass durch die Befragung aller Haushaltsmitglieder Informationen über beide Ehepartner verfügbar sind.

An dieser Stelle werden alle im SOEP enthaltenen Ehen im Zeitraum von 1985 bis 2010 analysiert, bei denen das Jahr der Eheschließung, das Haushaltseinkommen, das Vorhandensein von Kindern im Haushalt sowie der Erwerbsstatus beider Ehepartner bekannt sind. Insgesamt sind 15.066 Ehen im Zeitraum von 1985 bis $2010 \mathrm{im}$ SOEP erfasst, für die beide Ehepartner identifizierbar sind. Von diesen Ehen wurden 268 Ehen aufgrund von fehlendem oder zu geringem Heiratsalter (unter 16 Jahre) ausgeschlossen. Wegen fehlenden Werten bei der Berufsbildung bei einem Ehepartner oder bei beiden Ehepartnern fallen 974 Ehen aus der Analyse. Des Weiteren entfallen 774 Ehen aufgrund von fehlenden Werten zum Erwerbsstatus beider Ehepartner sowie 773 Ehen aufgrund von fehlenden Werten beim Nettoäquivalenzeinkommen. Insgesamt entfallen 2.578 Ehen aufgrund von einem oder mehreren fehlenden Werten. Somit stehen für die Analysen 12.488 Ehen mit 105.788 Ehejahren zur Verfügung.

Die zentrale unabhängige Variable, der Erwerbsstatus, wird unterschieden zwischen arbeitslos, erwerbstätig (Voll- und Teilzeitbeschäftigung inklusive Minijob, nebenberufliche Tätigkeit, Kurzarbeit, Wehr- und Zivildienst, betriebliche Aus- und Fortbildung) und nicht erwerbstätig (in Rente, Hausfrau, -mann, in Schule, in Hochschule, Mutterschaftsurlaub).

Für differenziertere Analysen werden die Arbeitslosen zudem nach der Dauer ihrer Arbeitslosigkeit in drei Gruppen eingeteilt: kurzzeitige Arbeitslosigkeit: 1 bis 12 Monate, längere Arbeitslosigkeit: 13 bis 24 Monate sowie Langzeitarbeitslosigkeit: 25 und mehr Monate. Die Einteilung der Dauer der Arbeitslosigkeit erfolgt durch die Anzahl der Monate, die eine Person zu Ende des Vorjahres ohne Unterbrechung arbeitslos war. ${ }^{3}$ Auch die Einteilung in die Gruppen erwerbstätig und nicht erwerbstätig wird auf Grundlage des Erwerbsstatus zum Ende des Vorjahres vorgenommen. Durch diese Operationalisierung ist die Kausalitätsrichtung eindeutig. Die Möglichkeit, dass eine Trennung der Auslöser von Arbeitslosigkeit ist, wird somit ausgeschlossen. Allerdings kommt es dadurch zu einer Unschärfe, die darin besteht, dass eine Person, die zum Ende des Vorjahres arbeitslos ist - und damit in die Kategorie der Arbeitslosen fällt -, zum Zeitpunkt der Trennung unter Umständen bereits wieder erwerbstätig sein kann. Um zu überprüfen, inwieweit mögliche Effekte der Arbeitslosigkeit auf den Einkommensverlust zurückzuführen sind, wird neben dem Erwerbsstatus auch das monatliche Nettoäquivalenzeinkommen des Vorjahres (in 1.000 Euro) berücksichtigt. Aus bisherigen Untersuchungen geht hervor, dass das Trennungsrisiko mit höherem Haushaltseinkommen sinkt (Lewin 2005; Conger et al. 1990;

3 Dadurch wird die Dauer der Arbeitslosigkeit auch auf null Monate gesetzt, sobald eine Person nur einen Monat nicht arbeitslos war. Somit können „faktisch“ Langzeitarbeitslose, die gelegentlich kurzzeitig eine Arbeitsstelle haben, in die Kategorie der seit kurzem Arbeitslosen fallen. 
White 1990). ${ }^{4}$ Wenn der Arbeitslosigkeitseffekt ausschließlich auf den Einkommensverlust zurückzuführen ist, also eigentlich ein Einkommenseffekt ist, dann müsste der Effekt der Arbeitslosigkeit unter Kontrolle des Einkommens komplett verschwinden.

Da sowohl der Erwerbsstatus als auch das Trennungsrisiko vom Bildungsniveau beeinflusst sein können (z.B. Babka von Gostomski et al. 1998), wird für beide Ehepartner der höchste berufliche Bildungsabschluss als Kontrollvariable in die Analyse mit aufgenommen. ${ }^{5}$ Weiterhin wird das Vorhandensein von Kindern im Haushalt berücksichtigt, da Kinder, die im elterlichen Haushalt wohnen, stabilisierend auf Ehen wirken (z.B. Klein/Rapp 2010). Dieser stabilisierende Einfluss nimmt jedoch mit zunehmendem Alter der Kinder ab (Böttcher 2006; Lyngstad 2004; Rapp 2008; Stauder 2006). Dem wird durch Gruppierung nach dem Alter des jüngsten Kindes im Haushalt Rechnung getragen. Als weitere Kontrollvariablen werden das Heiratsalter der Frau, die Ehedauer (siehe unten) sowie die Information, ob es für beide Ehepartner die erste Ehe ist, aufgenommen. Zudem wird das Kalenderjahr anhand von drei Kategorien abgebildet, die die 1980er, 1990er und 2000er Jahre repräsentieren.

Von den 12.488 untersuchten Ehen enden 940 im Untersuchungszeitraum mit einer Trennung. Den Berechnungen liegen allerdings nur 871 Trennungsereignisse zugrunde, da für 69 Ehen, die mit einer Trennung enden, die Informationen im letzten Ehejahr unvollständig sind. Einen Überblick über die Stichprobe gibt Tabelle 1.

Als Analyseverfahren wird die Ereignisdatenanalyse unter Verwendung des generalisierten Sichelmodells angewendet, wie es von Klein (2003: 512) vorgeschlagen wurde, um einen zu Beginn ansteigenden und später abfallenden Verlauf der Eintrittswahrscheinlichkeit eines Ereignisses darzustellen. Dies entspricht dem typischen Verlauf der Trennungsrate von Ehen (vgl. Rapp 2008) und wird durch folgende Formel beschrieben:

$$
\ln h_{i}(t)=a+b t+c \ln t+\sum_{j} \alpha_{j} x_{i j}+\sum_{k} \beta_{k} u_{i k}(t)
$$

Dabei beschreibt $\ln h_{i}(t)$ die logarithmierte Trennungsrate zum Zeitpunkt $t$ seit der Eheschließung. Bei der Berechnung mit der Methode des Episodensplittings (vgl. Blossfeld et al. 1986: 193 ff.) werden einjährige Zeitintervalle $i$ verwendet. ${ }^{6}$ Die Episoden werden hierbei nach dem Kalenderjahr aufgeteilt. Das Jahr der Heirat zählt als erstes Ehejahr. Mit $x_{i j}$ werden die zeitunabhängigen Variablen, wie z.B. das Heiratsalter, und mit $u_{i k}(t)$ die zeitabhängigen Variablen, wie z.B. das Einkommen, bezeichnet. Dabei steht $j$ bzw. $k$ für die 1 bis $n$ zeitunveränderlichen bzw. zeitveränderlichen Variablen. Während das zugrundeliegende Exponentialmodell eine zeitkonstante Rate impliziert, wird durch die Aufnahme von $t$ sowie $\ln t$ der zuerst ansteigende und später abfallende Verlauf der Trennungsrate modelliert. Da eine Person in den Daten mit mehreren Ehen vertreten sein kann, werden robuste Standardfehler berechnet. ${ }^{7}$

4 Betrachtet man nur das Einkommen der Frau, so zeigt sich jedoch, dass mit steigendem Einkommen auch das Trennungsrisiko steigt (u.a. Chan/Halpin 2002; Böttcher 2006; South 2001; Rapp 2008).

5 Um Fallzahlverluste zu vermeiden ist die Berufsbildung nicht als zeitabhängige Variable in den Analysen enthalten, sondern es wird der höchste Abschluss berücksichtigt, auch dann, wenn in einzelnen Jahren fehlende Werte vorliegen.

6 Auch dem Heirats- und Trennungsjahr wird eine Dauer von einem Jahr zugewiesen.

7 Die Berechnungen wurden mit der Statistiksoftware Stata (Version 12.1) und dem Kommando streg durchgeführt. 
Tabelle 1: Beschreibung der Stichprobe

\begin{tabular}{|c|c|c|c|}
\hline & & Ehen & Ehejahre \\
\hline \multicolumn{2}{|c|}{ Metrische Variablen } & \multicolumn{2}{|c|}{ Mittelwerte } \\
\hline \multicolumn{4}{|c|}{ Zeitunabhängig } \\
\hline \multicolumn{2}{|c|}{ Heiratsalter der Frau } & 25,9 & 25,1 \\
\hline \multicolumn{2}{|c|}{ Heiratsjahr } & 1978,2 & 1976,1 \\
\hline \multicolumn{2}{|c|}{ Ehejahr bei Trennung oder Zensierung } & 26,4 & \\
\hline \multicolumn{4}{|c|}{ Zeitabhängig } \\
\hline \multicolumn{2}{|c|}{ Nettoäquivalenzeinkommen in 1.000 Euro } & & 1,592 \\
\hline \multicolumn{2}{|c|}{ Kategoriale Variable } & \multicolumn{2}{|c|}{ Anzahl } \\
\hline \multicolumn{4}{|c|}{ Zeitunabhängig } \\
\hline \multicolumn{2}{|c|}{ Erstehe für beide Ehepartner } & 10.333 & 89.676 \\
\hline \multicolumn{2}{|c|}{ Zweitehe für mindestens einen Partner } & 2.155 & 16.112 \\
\hline \multicolumn{2}{|c|}{ Frau hat Hochschulabschluss } & 1.712 & 12.784 \\
\hline \multicolumn{2}{|c|}{ Frau hat Berufsabschluss } & 7.910 & 68.206 \\
\hline \multicolumn{2}{|c|}{ Frau hat keinen Berufsabschluss } & 2.866 & 24.798 \\
\hline \multicolumn{2}{|c|}{ Mann hat Hochschulabschluss } & 2.770 & 21.902 \\
\hline \multicolumn{2}{|c|}{ Mann hat Berufsabschluss } & 8.362 & 72.360 \\
\hline \multicolumn{2}{|c|}{ Mann hat keinen Berufsabschluss } & 1.356 & 11.526 \\
\hline \multicolumn{4}{|c|}{ Zeitabhängig } \\
\hline \multicolumn{2}{|c|}{ Jahr 1985-1989 } & & 14.513 \\
\hline \multicolumn{2}{|c|}{ Jahr 1990-1999 } & & 33.251 \\
\hline \multicolumn{2}{|c|}{ Jahr 2000-2010 } & & 58.024 \\
\hline \multicolumn{2}{|c|}{ Kein Kind unter 16 Jahren im Haushalt } & & 62.403 \\
\hline \multicolumn{2}{|c|}{ Kind unter 4 Jahren im Haushalt } & & 14.853 \\
\hline \multicolumn{2}{|c|}{ Kind von 4 bis unter 8 Jahren im Haushalt } & & 10.060 \\
\hline \multicolumn{2}{|c|}{ Kind von 8 bis unter 12 Jahren im Haushalt } & & 9.306 \\
\hline Kind von 12 & unter 16 Jahren im Haushalt & & 9.166 \\
\hline Frau ist erw & stätig & & 44.458 \\
\hline Frau ist nich & rwerbstätig & & 56.411 \\
\hline Frau ist arbe & & & 4.919 \\
\hline davon & 1 bis 12 Monate arbeitslos & & 2.677 \\
\hline & 13 bis 24 Monate arbeitslos & & 1.073 \\
\hline & mehr als 24 Monate arbeitslos & & 1.169 \\
\hline Mann ist erv & bstätig & & 70.676 \\
\hline Mann ist nic & erwerbstätig & & 30.093 \\
\hline Mann ist arb & slos & & 5.019 \\
\hline davon & 1 bis 12 Monate arbeitslos & & 2.644 \\
\hline & 13 bis 24 Monate arbeitslos & & 1.049 \\
\hline & mehr als 24 Monate arbeitslos & & 1.326 \\
\hline Fallzahl & & 12.488 & 105.788 \\
\hline
\end{tabular}

Quelle: SOEP 1984-2010; eigene Berechnungen 


\section{Ergebnisse}

Um das Trennungsrisiko bei Arbeitslosigkeit eines Ehepartners zu bestimmen, werden ereignisanalytische Regressionsmodelle berechnet. Die Koeffizienten $\beta$ können durch die Transformation $e^{\beta}$ in relative Risiken umgerechnet werden. Diese sind in der Tabelle 2 dargestellt und können folgendermaßen interpretiert werden: Ein Wert größer 1 zeigt eine Risikosteigerung, ein Wert kleiner 1 eine Risikosenkung an. Der Wert 0,72 für die Nichterwerbstätigkeit der Frau in Modell 1 gibt beispielsweise an, dass das Trennungsrisiko bei Nichterwerbstätigkeit der Frau im Vergleich zu Ehen mit erwerbstätiger Frau, der Referenzkategorie, um den Faktor 0,72 (bzw. um 28\%) geringer ist.

Tabelle 2: Effekte des Erwerbsstatus und weitere Determinanten des Trennungsrisikos (relative Risiken; robuste Standardfehler in Klammer)

\begin{tabular}{|c|c|c|c|c|c|}
\hline & Modell 1 & Modell 2 & Modell 3 & Modell 4 & Modell 5 \\
\hline \multirow[t]{2}{*}{ Konstante - Parameter a } & $0,01^{* * *}$ & $0,06^{* * *}$ & $0,05^{* * *}$ & $0,05^{\star * *}$ & $0,06^{\star * *}$ \\
\hline & $(0,00)$ & $(0,02)$ & $(0,02)$ & $(0,02)$ & $(0,02)$ \\
\hline \multirow[t]{2}{*}{ Ehejahr - Parameter b } & $0,86^{\star * *}$ & $0,85^{\star \star *}$ & $0,85^{\star \star \star}$ & $0,85^{\star * \star}$ & $0,85^{\star \star *}$ \\
\hline & $(0,01)$ & $(0,01)$ & $(0,01)$ & $(0,01)$ & $(0,01)$ \\
\hline \multirow[t]{2}{*}{ In (Ehejahr) - Parameter c } & $2,68^{\star * *}$ & $2,62^{\star \star *}$ & $2,62^{* * *}$ & $2,62^{\star * *}$ & $2,63^{\star * *}$ \\
\hline & $(0,27)$ & $(0,30)$ & $(0,30)$ & $(0,30)$ & $(0,30)$ \\
\hline \multirow[t]{2}{*}{ Frau ist nicht erwerbstätig ${ }^{1}$} & $0,72^{* * *}$ & $0,78^{* * *}$ & $0,78^{* *}$ & $0,78^{\star *}$ & $0,59^{* *}$ \\
\hline & $(0,05)$ & $(0,06)$ & $(0,06)$ & $(0,06)$ & $0,11)$ \\
\hline \multirow[t]{2}{*}{ Frau ist arbeitslos ${ }^{1}$} & 1,21 & 1,18 & 1,19 & & 0,71 \\
\hline & $(0,16)$ & $(0,16)$ & $(0,16)$ & & $(0,37)$ \\
\hline \multirow[t]{2}{*}{ Mann ist nicht erwerbstätig ${ }^{1}$} & $0,73^{*}$ & 0,91 & 0,92 & 0,92 & 0,83 \\
\hline & $(0,10)$ & $(0,13)$ & $(0,13)$ & $(0,13)$ & $(0,38)$ \\
\hline \multirow[t]{2}{*}{ Mann ist arbeitslos ${ }^{1}$} & $1,79^{* * *}$ & $1,70^{* * *}$ & $1.71^{\star * *}$ & & $3,08^{\star * *}$ \\
\hline & $(0,22)$ & $(0,21)$ & $(0,21)$ & & $(0,98)$ \\
\hline \multirow[t]{2}{*}{ Heiratsalter der Frau } & & $0,95^{\star * *}$ & $0,95^{\star \star *}$ & $0,05^{\star \star *}$ & $0,95^{\star \star *}$ \\
\hline & & $(0,01)$ & $(0,01)$ & $(0,01)$ & $(0,01)$ \\
\hline \multirow[t]{2}{*}{ Jahr $1990-1999^{2}$} & & $1,25^{\star}$ & $1,24+$ & $1,24+$ & 1,10 \\
\hline & & $(0,14)$ & $(0,14)$ & $(0,14)$ & $(0,18)$ \\
\hline \multirow[t]{2}{*}{ Jahr $2000-2010^{2}$} & & $1,33^{*}$ & $1,31^{*}$ & $1,32^{*}$ & 1,16 \\
\hline & & $(0,15)$ & $(0,15)$ & $(0,15)$ & $(0,18)$ \\
\hline \multirow[t]{2}{*}{ Erstehe für beide Ehepartner ${ }^{3}$} & & $0,50^{* * *}$ & $0,50^{* * *}$ & $0,50^{* * *}$ & $0,50^{* * *}$ \\
\hline & & $(0,05)$ & $(0,05)$ & $(0,05)$ & $(0,05)$ \\
\hline \multirow[t]{2}{*}{ Kind unter 4 Jahre im Haushalt ${ }^{4}$} & & 0,88 & 0,89 & 0,89 & 0,89 \\
\hline & & $(0,09)$ & $(0,10)$ & $(0,10)$ & $(0,09)$ \\
\hline \multirow[t]{2}{*}{ Kind von 4 bis unter 8 Jahre im Haushalt ${ }^{4}$} & & 0,90 & 0,91 & 0,91 & 0,90 \\
\hline & & $(0,10)$ & $(0,11)$ & $(0,11)$ & $(0,11)$ \\
\hline \multirow[t]{2}{*}{ Kind von 8 bis unter 12 Jahre in Haushalt ${ }^{4}$} & & 1,18 & 1,19 & 1,19 & 1,19 \\
\hline & & $(0,14)$ & $(0,14)$ & $(0,14)$ & $(0,14)$ \\
\hline \multirow[t]{2}{*}{ Kind von 12 bis unter 16 Jahre im Haushalt ${ }^{4}$} & & $1,34^{*}$ & $1,36^{*}$ & $1,36^{*}$ & $1,36^{*}$ \\
\hline & & $(0,16)$ & $(0,17)$ & $(0,17)$ & $(0,17)$ \\
\hline \multirow[t]{2}{*}{ Frau hat Berufsabschluss ${ }^{5}$} & & 1,05 & 1,04 & 1,04 & 1,05 \\
\hline & & $(0,10)$ & $(0,10)$ & $(0,10)$ & $(0,10)$ \\
\hline \multirow[t]{2}{*}{ Frau hat Hochschulabschluss ${ }^{5}$} & & 1,16 & 1,15 & 1.15 & 1,15 \\
\hline & & $(0,17)$ & $(0,16)$ & $(0,16)$ & $(0,17)$ \\
\hline \multirow[t]{2}{*}{ Mann hat Berufsabschluss ${ }^{5}$} & & 1,04 & 1,04 & 1,04 & 1,05 \\
\hline & & $(0,12)$ & $(0,12)$ & $(0,12)$ & $(0,12)$ \\
\hline
\end{tabular}




\begin{tabular}{|c|c|c|c|c|c|}
\hline & Modell 1 & Modell 2 & Modell 3 & Modell 4 & Modell 5 \\
\hline \multirow[t]{2}{*}{ Mann hat Hochschulabschluss ${ }^{5}$} & & 0,85 & 0,84 & 0,84 & 0,84 \\
\hline & & $(0,13)$ & $(0,13)$ & $(0,13)$ & $(0,13)$ \\
\hline \multirow[t]{2}{*}{ Nettoäquivalenzeinkommen in 1.000 Euro } & & & 1,03 & 1,03 & 1,03 \\
\hline & & & $(0,02)$ & $(0,02)$ & $(0,02)$ \\
\hline \multirow[t]{2}{*}{ Frau ist 1 bis 12 Monate arbeitslos ${ }^{1}$} & & & & 1,23 & \\
\hline & & & & $(0,19)$ & \\
\hline \multirow[t]{2}{*}{ Frau ist 13 bis 24 Monate arbeitslos ${ }^{1}$} & & & & 1,29 & \\
\hline & & & & $(0,35)$ & \\
\hline \multirow[t]{2}{*}{ Frau ist mehr als 24 Monate arbeitslos ${ }^{1}$} & & & & 0,96 & \\
\hline & & & & $(0,32)$ & \\
\hline \multirow[t]{2}{*}{ Mann ist 1 bis 12 Monate arbeitslos ${ }^{1}$} & & & & $1,72^{\star \star *}$ & \\
\hline & & & & $(0,26)$ & \\
\hline \multirow[t]{2}{*}{ Mann ist 13 bis 24 Monate arbeitslos ${ }^{1}$} & & & & $1,92^{* *}$ & \\
\hline & & & & $(0,47)$ & \\
\hline \multirow[t]{2}{*}{ Mann ist mehr als 24 Monate arbeitslos ${ }^{1}$} & & & & $1,56+$ & \\
\hline & & & & $(0,42)$ & \\
\hline \multirow[t]{2}{*}{ Frau ist nicht erwerbstätig ${ }^{1}$ * Jahr $1990-1999^{2}$} & & & & & 1,30 \\
\hline & & & & & $(0,30)$ \\
\hline \multirow[t]{2}{*}{ Frau ist nicht erwerbstätig ${ }^{1}{ }^{*} 2000-2010^{2}$} & & & & & $1,45+$ \\
\hline & & & & & $(0,32)$ \\
\hline \multirow[t]{2}{*}{ Frau ist arbeitslos ${ }^{1} * 1990-1999^{2}$} & & & & & 1,69 \\
\hline & & & & & $(0,94)$ \\
\hline \multirow[t]{2}{*}{ Frau ist arbeitslos ${ }^{1} * 2000-2010^{2}$} & & & & & 1,87 \\
\hline & & & & & $(1,03)$ \\
\hline \multirow[t]{2}{*}{ Mann ist nicht erwerbstätig ${ }^{1}$ * Jahr $1990-1999^{2}$} & & & & & 1,27 \\
\hline & & & & & $(0,64)$ \\
\hline \multirow[t]{2}{*}{ Mann ist nicht erwerbstätig ${ }^{1}$ * Jahr $2000-2010^{2}$} & & & & & 1,01 \\
\hline & & & & & $(0,50)$ \\
\hline \multirow[t]{2}{*}{ Mann ist arbeitslos ${ }^{1}$ * Jahr $1990-1999^{2}$} & & & & & 0,60 \\
\hline & & & & & $(023)$ \\
\hline \multirow[t]{2}{*}{ Mann ist arbeitslos ${ }^{1}$ Jahr $2000-2010^{2}$} & & & & & $0,46^{*}$ \\
\hline & & & & & $(0,17)$ \\
\hline Ereignisse & 871 & 871 & 871 & 871 & 871 \\
\hline Episoden & 105.788 & 105.788 & 105,788 & 105.788 & 105.788 \\
\hline Log-Pseudolikelihood & -4.659 & -4.607 & -4.607 & -4.606 & -4.603 \\
\hline
\end{tabular}

*** $\mathrm{p}<0,001 ; * * \mathrm{p}<0,01 ; * \mathrm{p}<0,05 ;+\mathrm{p}<0,1$

1) Referenzkategorie: Frau bzw. Mann ist erwerbstätig

2) Referenzkategorie: Jahr 1985-1989

3) Referenzkategorie: Zweitehe für mindestens einen Partner

4) Referenzkategorie: Kein Kind unter 16 Jahren im Haushalt

5) Referenzkategorie: Frau bzw. Mann hat keinen Berufsabschluss

Quelle: SOEP 1984-2010; eigene Berechnungen

In Modell 1 sind neben den für das Sichelmodell nötigen Parametern - das Ehejahr und das logarithmierte Ehejahr - nur die Dummyvariablen des Erwerbsstatus beider Ehepartner enthalten. Als Ergebnis zeigt sich, dass Arbeitslosigkeit eines Ehepartners mit einem erhöhten Trennungsrisiko einhergeht. Ist der Ehemann arbeitslos, so liegt das Trennungsrisiko ca. 79\% über dem eines Paares mit erwerbstätigem Mann. Auch Arbeitslosigkeit der Frau hat einen destabilisierenden Effekt auf Ehen, welcher jedoch mit einem relativen Risiko von 1,21 schwächer ausfällt als bei Männern und nicht signifikant ist. Sowohl bei 
Männern als auch bei Frauen geht Nichterwerbstätigkeit mit einem im Vergleich zur Erwerbstätigkeit geringeren Trennungsrisiko einher.

Während Modell 1 den Zusammenhang zwischen Arbeitslosigkeit und Trennungsrisiko beschreibt, zielt Modell 2 darauf ab, den Einfluss von Arbeitslosigkeit auf das Trennungsrisiko zu testen. Geprüft wird, ob Arbeitslosigkeit das Trennungsrisiko erhöht (Hypothese 1a) und ob dies in stärkerem Maße für Männer der Fall ist als für Frauen (Hypothese 2). Im Unterschied zu Modell 1 sind zusätzlich die berufliche Ausbildung von beiden Partnern, das Alter des jüngsten Kindes im Haushalt, das Heiratsalter der Frau, die Kalenderzeit, sowie ob es sich für beide Partner um die erste Ehe handelt, kontrolliert. ${ }^{8}$ Dadurch wird der destabilisierende Effekt von Arbeitslosigkeit des Mannes geringfügig abgeschwächt, bleibt jedoch mit einem - im Vergleich zu Ehen mit erwerbstätigem Mann - um ca. 70\% erhöhten Trennungsrisiko sehr stark. Die stabilisierende Wirkung von Nichterwerbstätigkeit der Ehefrau nimmt etwas ab, bleibt jedoch signifikant. Im Gegensatz dazu wird der stabilisierende Einfluss der Nichterwerbstätigkeit des Mannes unter Kontrolle weiterer Einflussfaktoren nicht bestätigt.

Um zu überprüfen, ob der destabilisierende Effekt von Arbeitslosigkeit auf die Ehebeziehung zu einem Teil auf den mit Arbeitslosigkeit einhergehenden Einkommensverlust zurückzuführen ist (Hypothese 3), wird in Modell 3 zusätzlich das Haushaltseinkommen der Ehepaare berücksichtigt. Für das Einkommen kann kein Einfluss auf das Trennungsrisiko nachgewiesen werden, und die Effekte der Nichterwerbstätigkeit und der Arbeitslosigkeit der Frau und des Mannes bleiben praktisch unverändert.

In Modell 4 wird geprüft, ob sich der Effekt von Arbeitslosigkeit mit zunehmender Dauer ändert (Hypothesen 4a und 4b). Hierzu wird unterschieden, ob Arbeitslosigkeit seit 1-12 Monaten, 13-24 Monaten oder seit mehr als 24 Monaten besteht. Für Frauen zeigen sich für keine der drei Arbeitslosigkeitsdauern signifikante Effekte, wohingegen für Männer sowohl eine kürzere als auch eine mittlere Arbeitslosigkeitsdauer ein erhöhtes Trennungsrisiko zur Folge hat. Dabei ist das Trennungsrisiko bei einer Arbeitslosigkeitsdauer zwischen einem und zwei Jahren am höchsten. Für langzeitarbeitslose Männer (über zwei Jahre arbeitslos) ist das Trennungsrisiko nur schwach signifikant erhöht.

Um zu überprüfen, ob sich der Arbeitslosigkeitseffekt im Zeitverlauf für Männer abgeschwächt und für Frauen verstärkt und somit angeglichen hat (Hypothese 5), werden in Modell 5 Interaktionen zwischen dem Erwerbsstatus der Ehepartner und dem Kalenderjahr in die Berechnung aufgenommen. Für die Arbeitslosigkeit des Mannes zeigt sich eine Abschwächung des Effekts im untersuchten Zeitraum: Der destabilisierende Effekt ist in den Jahren 2000 bis 2010 signifikant niedriger als in den Jahren 1985 bis 1989. Für Frauen zeigt sich keine signifikante Veränderung des Arbeitslosigkeitseffektes im Zeitverlauf. Jedoch reduziert sich für Frauen der stabilisierende Effekt einer Nichterwerbstätigkeit von den 1980er Jahren bis zu den 2000er Jahren, der diesbezügliche Interaktionseffekt ist allerdings nur auf dem 10\%-Niveau statistisch abgesichert.

8 Durch die Kontrollvariablen werden einige bekannte Effekte auf die Ehestabilität bestätigt. Das Trennungsrisiko ist geringer, wenn es für beide Partner die erste Ehe ist. Auch ein höheres Heiratsalter wirkt stabilisierend. Zudem kann im Zeitverlauf sowie mit steigendem Alter der Kinder ein ansteigendes Trennungsrisiko beobachtet werden. Für die Berufsbildung lassen sich keine Einflüsse auf die Stabilität der Ehe nachweisen. 


\section{Diskussion}

Die vorliegende Studie zeigt, dass Arbeitslosigkeit mit einem erhöhten Trennungsrisiko einhergeht. Dies trifft in stärkerem Maße für Männer zu als für Frauen, bei denen der Zusammenhang schwächer ausgeprägt und nicht signifikant ist. Der destabilisierende Effekt von Arbeitslosigkeit bleibt auch unter Kontrolle von Drittvariablen bestehen, für die in Betracht zu ziehen ist, dass diese Faktoren sowohl (und voneinander unabhängig) das Trennungsrisiko beeinflussen als auch das Risiko, arbeitslos zu sein. Das höhere Trennungsrisiko von Arbeitslosen lässt sich folglich zum Beispiel nicht dadurch erklären, dass sich Arbeitslose hinsichtlich ihrer beruflichen Ausbildung oder hinsichtlich ihres Heiratsalters von Erwerbstätigen unterscheiden. Die Ergebnisse stehen im Einklang mit den theoretischen Erwartungen der Hypothesen 1a (Arbeitslosigkeit erhöht das Trennungsrisiko) und 2 (die Erhöhung fällt stärker aus wenn der Ehemann arbeitslos ist) und stimmen zudem mit früheren empirischen Befunden überein. Nicht auszuschließen ist allerdings, dass andere Drittvariablen, die im Rahmen dieser Untersuchung nicht kontrolliert werden konnten (z.B. Substanzmissbrauch), ursächlich für die beobachteten Effekte sind.

In der vorliegenden Studie wurde des Weiteren untersucht, ob der destabilisierende Effekt von Arbeitslosigkeit auf die Ehe zu einem Teil auf den mit Arbeitslosigkeit einhergehenden Einkommensverlust zurückzuführen ist (Hypothese 3). Entgegen den Erwartungen zeigt sich, dass ein Einkommensverlust aufgrund von Arbeitslosigkeit keine Ursache für das höhere Trennungsrisiko im Falle von Arbeitslosigkeit ist. Denn der Arbeitslosigkeitseffekt wird auch nach Kontrolle des Einkommens nicht kleiner. Dies bedeutet, dass andere Faktoren als ein niedrigeres Einkommen den ungünstigen Einfluss von Arbeitslosigkeit auf das Trennungsrisiko vermitteln.

Ein weiteres Ergebnis der vorliegenden Studie ist, dass sowohl bei kurzer als auch bei längerer Dauer der Arbeitslosigkeit des Mannes ein erhöhtes Trennungsrisiko zu beobachten ist, wobei das Trennungsrisiko bei einer mittleren Dauer von einem bis zwei Jahren am höchsten ist. Die beobachteten Unterschiede im Trennungsrisiko nach der Dauer der Arbeitslosigkeit des Mannes sind allerdings zu gering, um folgern zu können, dass das Trennungsrisiko mit zunehmender Arbeitslosigkeitsdauer erst zu- und dann wieder abnimmt. Somit kann weder die Hypothese 4a, wonach der Effekt von Arbeitslosigkeit auf das Trennungsrisiko mit zunehmender Dauer der Arbeitslosigkeit abnimmt, noch Hypothese $4 \mathrm{~b}$, wonach der risikosteigernde Effekt zunimmt, uneingeschränkt bestätigt werden. Um die Frage beantworten zu können, ob die Dauer der Arbeitslosigkeit das Trennungsrisiko beeinflusst, sind Untersuchungen notwendig, die sich auf größere Fallzahlen stützen können als die vorliegende Studie.

Schließlich zeigt die vorliegende Untersuchung, dass der destabilisierende Effekt der Arbeitslosigkeit des Mannes im Zeitraum von 2000 bis 2010 schwächer ausfällt als in den Jahren 1985 bis 1989. Hypothese 5 wird damit nur teilweise bestätigt. Für Frauen zeigt sich in diesem Zeitraum keine Veränderung des Arbeitslosigkeitseffekts. Allerdings ist für Frauen der stabilisierende Effekt einer Nichterwerbstätigkeit in der Zeit von 2000 bis 2010 geringer als noch Ende der 1980er Jahre.

Diese beiden Ergebnisse - also die Abschwächung des ungünstigen Arbeitslosigkeitseffekts bei Männern sowie der schwächer werdende stabilisierende Effekt der Nichterwerbstätigkeit der Frau - stehen möglicherweise damit in Zusammenhang, dass die tradi- 
tionelle Arbeitsteilung, bei der nur der Mann erwerbstätig oder zumindest der Haupternährer ist, faktisch an Bedeutung verloren hat. Aus diesem Grund haben vermutlich die finanziellen und möglicherweise auch die psychosozialen Belastungen für die Ehepartner im Falle einer Arbeitslosigkeit des Mannes abgenommen. Um zu klären, ob es sich bei der in dieser Untersuchung beobachteten Angleichung bei den Erwerbseffekten von Männern und Frauen auf die Beziehungsstabilität um einen stabilen Trend handelt, und ob dieser z.B. in Perioden- oder in Kohorteneffekten begründet ist, sind allerdings weitere Untersuchungen notwendig.

\section{Literatur}

Amato, P. R. \& Beattie, B. (2011). Does the unemployment rate affect the divorce rate? An analysis of state data 1960-2005. Social Science Research, 40, 3, S.705-715.

Angell, R. C. (1936). The family encounters the depression. New York: Charles Scribner \& Sons.

Atkinson, T., Liem, R. \& Liem, J. H. (1986). The social costs of unemployment. Implications for social support. Journal of Health and Social Behavior, 27, 4, S. 317-331.

Aubry, T., Tefft, B. \& Kingsbury, N. (1990). Behavioral and psychological consequences of unemployment in blue-collar couples. Journal of Community Psychology, 18, 2, S. 99-109.

Babka von Gostomski, C., Hartmann, J. \& Kopp J. (1998). Sozialstrukturelle Bestimmungsgründe der Ehescheidung. Eine empirische Überprüfung einiger Hypothesen der Familienforschung. Zeitschrift für Soziologie der Erziehung und Sozialisation 18, 2, S. 117-133.

Bakke, E. W. (1940). Citizens without work. New York: Yale University Press.

Becker, G. S. (1993). A treatise on the family. Cambridge: Harvard University Press.

Becker, G. S., Landes, E. M. \& Michael, R. T. (1977). An economic analysis of marital instability. Journal of Political Economy, 85, 6, S. 1141-1187.

Bleich, C. \& Witte, E. H. (1992). Zu Veränderungen in der Paarbeziehung bei Erwerbslosigkeit des Mannes. Kölner Zeitschrift für Soziologie und Sozialpsychologie, 44, 4, S. 731-746.

Blossfeld, H.-P., Hamerle, A. \& Mayer, K. U. (1986). Ereignisanalyse. Statistische Theorie und Anwendung in den Wirtschafts- und Sozialwissenschaften. Frankfurt am Main \& New York: Campus Verlag.

BMAS - Bundesministerium für Arbeit und Soziales (2013). Lebenslagen in Deutschland. Vierter Armutsund Reichtumsbericht der Bundesregierung. Bonn: Bundesministerium für Arbeit und Soziales.

Böheim, R. \& Ermisch, J. (2001). Partnership dissolution in the UK - The role of economic circumstances. Oxford Bulletin of Economics and Statistics, 63, 2, S. 197-208.

Böttcher, K. (2006). Scheidung in Ost- und Westdeutschland. Der Einfluss der Frauenerwerbstätigkeit auf die Ehestabilität. Kölner Zeitschrift für Soziologie und Sozialpsychologie, 58, 4, S. 592-616.

Brinkerhoff, D. B. \& White, L. K. (1978). Marital satisfaction in an economically marginal population. Journal of Marriage and the Family, 40, 2, S. 259-267.

Brinkmann, C. (1976). Finanzielle und psycho-soziale Belastungen während der Arbeitslosigkeit. Mitteilungen aus der Arbeitsmarkt- und Berufsforschung, 9, 4, S. 397-413.

Brinkmann, C. (1984). Die individuellen Folgen langfristiger Arbeitslosigkeit. Ergebnisse einer repräsentativen Längsschnittuntersuchung. Mitteilungen aus der Arbeitsmarkt- und Berufsforschung, 17, 4, S. 454-473.

Cavan, R. S. (1959). Unemployment. Crisis of the common man. Marriage and Family Living, 21, 2, S. 139-146.

Cavan, R. S. \& Ranck, K. H. (1939). The family and the depression. Chicago: University of Chicago Press.

Chan, T. W. \& Halpin, B. (2002). Union dissolution in the United Kingdom. International Journal of Sociology, 32, 4, S. 76-93. 
Charles, K. K. \& Stephens M. (2004). Job displacement, disability, and divorce. Journal of Labor Economics, 22, 2, S. 489-522.

Conger, R. D., Elder Jr, G. H., Lorenz, F. O., Conger, K. J, Simons, R. L, Whitbeck, L. B., Huck, S. \& Melby, J. N. (1990). Linking economic hardship to marital quality and instability. Journal of Marriage and the Family, 52, 3, S. 643-656.

Cooke, L. P. \& Gash, V. (2010). Wives' part-time employment and marital stability in Great Britain, West Germany and the Unites States. Sociology, 44, 6, S. 1091-1108.

Dew, J. (2011). Financial issues and relationship outcomes among cohabiting individuals. Family Relations, 60, 2, S. 178-190.

Doiron, D. \& Mendolia, S. (2011). The impact of job loss on family dissolution. Journal of Population Economics, 25, 1, S. 367-398.

Eisenberg, P. \& Lazarsfeld, P. F. (1938). The psychological effects of unemployment. Psychological Bulletin, 35, 6, S. 358-391.

Eliason, M. (2012). Lost jobs, broken marriages. Journal of Population Economics, 25, 4, S. 1365-1397.

Esser, H. (2002). In guten wie in schlechten Tagen? Das Framing der Ehe und das Risiko zur Scheidung. Eine Anwendung und ein Test des Modells der Frame-Selektion. Kölner Zeitschrift für Soziologie und Sozialpsychologie, 54, 1, S. 27-63.

Gerlach, K. \& Stephan, G. (1996). A paper on unhappiness and unemployment in Germany. Economics Letters, 52, 3, S. 325-330.

Gerlach, K. \& Stephan, G. (2001). Lebenszufriedenheit und Erwerbsstatus: Ost- und Westdeutschland im Vergleich. Mitteilungen aus der Arbeitsmarkt- und Berufsforschung, 34, 4, S. 515-529.

Hansen, H.-T. (2005). Unemployment and marital dissolution. A panel data study of Norway. European Sociological Review, 21, 2, S. 135-148.

Hartley, J. (1987). Managerial unemployment. The wife's perspective and role, In: Fineman, S. \& Sinfield, A. (Hrsg.), Unemployment. Personal and social consequences. London: Tavistock (Social Science Paperbacks, 348), S. 118-137.

Hess, D., Hartenstein, W. \& Smid, M. (1991). Auswirkungen von Arbeitslosigkeit auf die Familie. Mitteilungen aus der Arbeitsmarkt- und Berufsforschung, 24, 1, S. 178-192.

Jalovaara, M. (2001). Socio-economic status and divorce in first marriages in Finland 1991-93. Population Studies, 55, 2, S. 119-133.

Jalovaara, M. (2003). The joint effects of marriage partners' socioeconomic positions on the risk of divorce. Demography, 40, 1, S. 67-81.

Jensen, P. \& Smith, N. (1990). Unemployment and marital dissolution. Journal of Population Economics, 3, 3, S. 215-229.

Kalmijn, M. (2007). Explaining cross-national differences in marriage, cohabitation, and divorce in Europe, 1990-2000. Population Studies, 61, 3, S. 243-263.

Kassenboehmer, S. C. \& Haisken-DeNew, J. P. (2009). You're fired! The causal negative effect of entry unemployment on life satisfaction. The Economic Journal, 119, 536, S. 448-462.

Kawata, Y. (2008). Does high unemployment rate result in a high divorce rate? A test for Japan. Revista de Economía del Rosario, 11, 2, S. 149-164.

Kessler, R. C., House, J. S. \& Turner, J. B. (1987). Unemployment and health in a community sample. Journal of Health and Social Behavior, 28, 1, S. 51-59.

Klein, T. (2003). Die Geburt von Kindern in paarbezogener Perspektive. Zeitschrift für Soziologie, 32, 6, S. 506-527.

Klein, T. \& Rapp, I. (2010). Der Einfluss des Auszugs von Kindern aus dem Elternhaus auf die Beziehungsstabilität der Eltern. Zeitschrift für Soziologie, 39, 2, S. 140-150.

Komarovsky, M. (1940). The unemployed man and his family. The effect of unemployment upon the status of the man in fifty-nine families. New York: Dryden Press.

Kraft, K. (2001). Unemployment and the seperation of married couples. KYKLOS, 54, 1, S. 67-88.

Lampard, R. (1994). An examination of the relationship between marital dissolution and unemployment, In: Gallie, D., Marsh, C. \& Vogler, C. (Hrsg.), Social change and the experience of unemployment. Oxford: Oxford University Press, S. 264-298. 
Larson, J. H. (1984). The effect of husband's unemployment on marital and family relations in bluecollar families. Family Relations, 33, 4, S. 503-511.

Levinger, G. (1976). A social psychological perspective on marital dissolution. Journal of Social Issues, 32,1, S. $21-47$

Levinger, G. (1982). A social exchange view on the dissolution of pair relationships, In: Nye, F. I. (Hrsg.), Family relationships. Rewards and costs. Beverly Hills: Sage, S. 97-121.

Lewin, A. C. (2005). The effect of economic stability among welfare recipients. Evaluation Review, 29, 3, S. 223-240.

Lewis, R. A. \& Spanier, G. B. (1979). Theorizing about the quality and stability of marriage, In: Burr, W., Hill, R., Nye, F. I. \& Reiss, I. (Hrsg.), Contemporary theories about the family. Volume 1. New York \& London: Free Press, S. 268-294.

Lewis, R. A. \& Spanier, G. B. (1982). Marital quality, marital stability, and social exchange, In: Nye, F. I. (Hrsg.), Family relationships. Rewards and costs. Beverly Hills: Sage, S. 49-65.

Lyngstad, T. H. (2004). The impact of parent's and spouses' education on divorce rates in Norway. Demographic Research, 10, 5, S. 121-142.

Nye, F. I. (Hrsg.) (1982). Family relationships. Rewards and costs. Beverly Hills: Sage.

OECD - Organization for Economic Co-operation and Development (2012). Unemployment rate. Employment and labour markets: Key tables from OECD, No. 1. Paris: Organization for Economic Cooperation and Development.

Powell, D.H. \& Driscoll, P. F. (1973). Middle-class professionals face unemployment. Society, 10, 2, S. 18-26.

Rapp, I. (2008). Wann werden Ehen getrennt? Der Einfluss der Ehedauer auf das Trennungsrisiko. Kölner Zeitschrift für Soziologie und Sozialpsychologie, 60, 3, S. 500-527.

Rege, M., Telle, K. \& Votruba, M. (2007). Plant closure and marital dissolution. Oslo: Statistics Norway, Research Department (Discussion Papers No. 514).

Root, K. (1984). The human response to plant closures. The ANNALS of the American Academy of Political and Social Science, 475, 1, S. 52-65.

Schindler, H. \& Wetzels, P. (1990). Familiensysteme in der Arbeitslosigkeit, In: Schindler, H., Wacker, A. \& Wetzels, P. (Hrsg.), Familienleben in der Arbeitslosigkeit. Ergebnisse neuerer europäischer Studien. Heidelberg: Roland Asanger, S. 43-73.

South, S. J. (2001). Time-dependent effects of wives' employment on marital dissolution. American Sociological Review, 66, 2, S. 226-245.

Starkey, J. L. (1996). Race differences in the effect of unemployment on marital instability: A socioeconomic analysis. Journal of Socio-Economics, 25, 6, S. 683-720.

Stauder, J. (2006). Die Verfügbarkeit partnerschaftlich gebundener Akteure für den Partnermarkt. Kölner Zeitschrift für Soziologie und Sozialpsychologie, 58, 4, S. 617-637.

Wagner, G. G., Göbel, J., Krause, P., Pischner, R. \& Sieber, I. (2008). Das Sozio-oekonomische Panel (SOEP): Multidisziplinäres Haushaltspanel und Kohortenstudie für Deutschland - Eine Einführung (für neue Datennutzer) mit einem Ausblick (für erfahrene Anwender). AStA Wirtschafts- und Sozialstatistisches Archiv, 2, 4, S. 301-328.

Weber, A., Hörmann, G. \& Heipertz, W. (2007). Arbeitslosigkeit und Gesundheit aus sozialmedizinischer Sicht. Deutsches Ärzteblatt, 104, 43, S. A2957-A2962.

White, L. K. (1990). Determinants of divorce. A review of research in the eighties. Journal of Marriage and the Family, 52, 4, S. 904-912.

Wilhelm, M. S. \& Ridley, C. A. (1988). Stress and unemployment in rural nonfarm couples: A study of hardships and coping resources. Family Relations, 37, 1, S. 50-54.

Winkelmann, L. \& Winkelmann, R. (1998). Why are the unemployed so unhappy? Evidence from panel data. Economica, 65, 257, S. 1-15.

Zvonkovic, A. M., Guss, T. \& Ladd, L. (1988). Making the most of job loss: Individual and marital features of underemployment. Family Relations, 37, 1, S. 56-61. 
Anschriften der Autoren/Addresses of the authors:

Fabio Franzese, M.A. (Korrespondenzautor/Corresponding author)

Munich Center for the Economics of Aging (MEA)

Max-Planck-Institut für Sozialrecht und Sozialpolitik

Amalienstraße 33

80799 München

Deutschland/Germany

Dr. Ingmar Rapp

Max-Weber-Institut für Soziologie der Universität Heidelberg,

Bergheimer Straße 58

69115 Heidelberg

Deutschland/Germany

E-Mail: franzese@mea.mpisoc.mpg.de ingmar.rapp@soziologie.uni-heidelberg.de 